“C 2019 IEEE. Personal use of this material is permitted. Permission from IEEE must be obtained for all other uses, in any current or future media, including reprinting/republishing this material for advertising or promotional purposes, creating new collective works, for resale or redistribution to servers or lists, or reuse of any copyrighted component of this work in other works." 


\section{DICE: Deep Intelligent Contextual Embedding for Twitter Sentiment Analysis}

\begin{abstract}
The sentiment analysis of social media-based short text (e.g., Twitter messages) is very valuable for many good reasons, explored increasingly in different communities such as text analysis, social media analysis, and recommendation. However, it is challenging as tweet-like social media text is often short, informal and noisy, and involves language ambiguity such as polysemy. The existing sentiment analysis approaches are mainly for document and clean textual data. Accordingly, we propose a Deep Intelligent Contextual Embedding (DICE), which enhances the tweet quality by handling noises within contexts, and then integrates four embeddings to involve polysemy in context, semantics, syntax, and sentiment knowledge of words in a tweet. DICE is then fed to a Bi-directional Long Short Term Memory (BiLSTM) network with attention to determine the sentiment of a tweet. The experimental results show that our model outperforms several baselines of both classic classifiers and combinations of various word embedding models in the sentiment analysis of airline-related tweets.
\end{abstract}

\section{INTRODUCTION}

Understanding the content and sentiment of information published online, including the social media platforms, where people share their opinions and views, is crucial from the perspective of improving the services, products, and recommendations for those users. Although many different approaches have been proposed, we are still not able to fully utilize the polysemy in the context, semantic information, sentiment, and syntax of the published information. This is especially vivid in the case of short statements and descriptions as tweets. With limited information available, e.g., tweets are limited to 140 characters, the analysis becomes very challenging.

Additionally, the language used on social media platforms and blogs is ubiquitous in nature as it is unstructured and very informal at times. Users write in their own words, and use abbreviations, different punctuations, incorrect spelling, emoticons, slang words, and URLs, etc. All of those language imperfections cause a lot of noise in the data, and one of the major challenges is to handle this unstructured and informal text by applying appropriate cleaning and pre-processing mechanisms.

Other than the noisy nature of tweets, utilizing the context of tweets in terms of polysemy, semantics, syntax and having sentiment knowledge of words are crucial for Twitter sentiment analysis. To represent the semantic information within tweets context, distributed word representation models like Word2Vec[16] and GloVe[19] have been used. Even though compelling improvements have been achieved by using distributed word representation in deep neural network models, there are still some limitations such as inability to identify and handling polysemy as well as noise within the context of
Example of word "Bad" in Tweets

@united Nope - still no one helped me. Giving up on united. \#badservice @USAirways Thank you!!! This whole crew has rocked through bad weather and diversion. Pilot keeping us well informed. \#customerservice

Example of word "Good" in Tweets

@united in the future when delay causes 15 hour wait (slept night in airport) ensuring seating choice for replacement flight would be good. @united you're good. Thank you!

Fig. 1. Words with different meanings and polarities in the context of tweets

tweets. Our research efforts should focus on making sure that learned representations: (i) capture polysemy in the context; (ii) represent complicated attributes of words usage including both semantics and syntax; and (iii) consider the sentiments of words.

Examples of polysemy and words with opposite polarity are shown in Fig. 1 where the meaning of words like 'good' and 'bad' changes according to its context which traditional word embeddings are unable to capture but assign the same representation of a word irrespective of its context and meaning. In addition to polysemy, traditional word embeddings fail to capture sentiment information of words like 'good' and 'bad' which results in similar word vector representations having the opposite polarities. Thus, ignoring polysemy within the context and sentiment polarity of words in a tweet reduces the performance of sentiment analysis.

In this research, we propose DICE (Deep Intelligent Contextualized Embedding) to solve the issues of polysemy, semantics, syntax and sentiment for a Twitter sentiment analysis. The input of DICE is fed to BiLSTM with attention for Twitter sentiment analysis. Also, our intelligent tweets pre-processor compliments our proposed model to learn better features by removing noise from unstructured and informal text.

The experiments are carried out on airline related tweets. The accuracy results for Twitter sentiment analysis, when using our proposed method, is higher than the state-of-the-art methods. Our contributions can be summarized as follows:

- An intelligent tweets pre-processor is designed to standardize the noisy nature of tweets by spell correction, sentiment aware tokenization, word segmentation and normalization.

- A Deep Intelligent Contextual Embedding (DICE) which addresses the language ambiguity and is devised to comprehensively capture polysemy in context, semantics, syntax and sentiment knowledge of words.

- Extensive experiments are conducted on several real- 


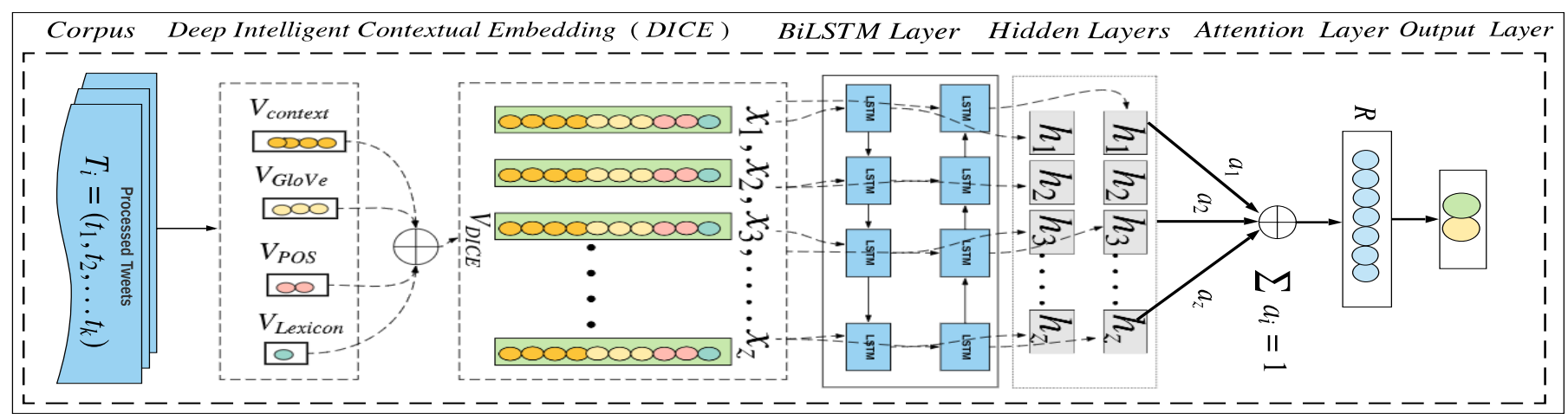

Fig. 2. DICE with BiLSTM and Attention layer

world datasets to evaluate above design. All the results prove that our model constantly outperforms other stateof-the-art methods.

The rest of the paper is organized as follows. Section II summarizes the relevant work. Section III describes the model architecture which includes our intelligent pre-processor, deep intelligent contextual embedding and deep neural network with attention. Section IV presents evaluation and analysis of model. Section V provides the conclusion of this research.

\section{RELATED WORK}

Sentiment analysis has attracted a lot of interest in the research community. Traditional methods for sentiment classification such as lexicon based methods [5] are simple, computationally economical and expendable but have some limitations such as their reliability on human efforts to label documents which is time consuming, have low coverage and not much effective in case of tweets where text is unstructured and informal. Several researchers claimed that using machine learning methods and hybrid of machine learning with lexicon based gives better performance [6].

Deep learning have played an important role in natural language processing (NLP). Bengio et al.[1] proposed a method which used neural network language model (NNLM) to learn word representations based on prior contexts of every word. After this breakthrough, different investigations were conducted in NLP using deep learning. Mikolov et al.[16] proposed continuous bag of words (CBOW) and skipgram models which uses a one layer architecture of word embeddings which was based on linear and local context which was solved by dependency based word embeddings and global vectors (GloVe)[19]. Jianqiang et al. [12] improved the accuracy results by using GloVe embedding with deep convolutional neural network (DCNN) for Twitter sentiment analysis, whereas Santos et al.[7] used character to sentence Convolutional Neural Network (CharSCNN) for sentiment analysis of short text which improved the accuracy results. All above methods ignore usage of polysemy in the context, Liu et al.[14] proposed context sensitive embeddings to overcome the issue of polysemy in general word embeddings which assigns one vector to each individual word. McCann et al. [15] proposed contextualized word vectors (CoVe) by computing contextualized representations using neural machine translation encoder. Most recently, Peters et al.[20] proposed deep contextual word representations for learning complex attributes of a word use in a context.

To integrate the sentiment information into traditional word embeddings, researchers proposed sentiment specific embeddings. Tang et al.[23] proposed several hybrid ranking models (HyRank) and developed sentiment embeddings based on $\mathrm{C} \& \mathrm{~W}$, which considers context and sentiment polarity of tweets.In $\mathrm{Yu}$ et al. [26] proposed sentiment embeddings by refining pre-trained embeddings $\operatorname{Re}\left({ }^{*}\right)$ using the intensity score of external knowledge resource. Razaeinia et al [21] proposed improved word vectors (IWV) by combining word embeddings, part of speech (POS) and combination of lexicons for sentiment analysis. Recently, Cambria et al.[2] proposed context embeddings for sentiment analysis by conceptual primitives from text and linked with commonsense concepts and named entities. Unlike above mentioned work, our proposed method is a context sensitive which considers complex attributes of words such as polysemy, semantics, syntax as well as sentiments of words for Twitter sentiment analysis.

\section{PROPOSED MODEL}

In this section we will describe our proposed model which is based on A ) Intelligent Pre-Processor, B) Deep Intelligent Contextual Embedding (DICE) C) Bi-directional Long Short Term Memory (BiLSTM) with Attention. The complete architecture of our proposed model is given in Fig.2. At input layer, our model gets an input of a processed tweet. Then in second layer, firstly, all words in a tweet are POS tagged and are assigned a vector. Secondly, using embeddings from language model, vector of each word is extracted which contains polysemy and syntax information and gives us a context embeddings. Thirdly, using GloVe embeddings, vector of words is created which captures word semantics information and the at next step, sentiment score of each word in a tweet is extracted from lexicons and lexicon vector is generated. All four vectors are then concatenated at third layer to produce 


\begin{tabular}{|c|c|}
\hline UnProcessed Tweets & Processed Tweets \\
\hline $\begin{array}{l}\text { @virginamerica why don't any of the pairings include red wine. Only white } \\
\text { is offered :( \#redwineisbetter } \\
\text { @united Hey so many tym changes for UA 1534. We going tonight or } \\
\text { what? MIA }\end{array}$ & $\begin{array}{l}\text { why do not any of the pairings include red wine only white is offered sad red } \\
\text { wine is better } \\
\text { hey so many time changes for ua } 1534 \text { we going tonight or what Missing In } \\
\text { Action. }\end{array}$ \\
\hline
\end{tabular}

Fig. 3. An Example our Tweets Pre-Processor which compliments our model to learn better features

our deep intelligent contextual embeddings (DICE) which is fed to BiLSTM with attention for tweets sentiment analysis. Below we describe each of the main components.

\section{A. Intelligent Pre-processor}

Our intelligent tweets pre-processor is designed to remove the noise from informal and unstructured tweets by correction of spelling mistakes, sentiment aware tokenization such as replacing emoticons and slangs with actual words and word segmentation of hashtag words in tweets which compliments our model to learn better features. For sentiment aware tokenization we benefited from Potts's tokenizer ${ }^{1}$ which is able to capture basic sentiment related expressions but our tokenizer is also able to identify more recent expressions and slangs being used in social media. For spell correction we borrowed idea proposed by Gimpel et al.[8] but we used Viterbi algorithm which is more effective in our case instead of metaphone algorithm. Lastly, we performed word segmentation to separate words in hashtags, normalized all words, removed punctuations, stops words, mentions (@), URLs and special characters. Examples of our Intelligent pre-processor is shown in Fig. 3.

\section{B. Deep Intelligent Contextual Embedding (DICE)}

Given a Tweet $T_{i}$ with a sequence of tokens $\left(t_{1}, t_{2}, t_{3}, \ldots t_{k}\right)$, where $i$ represents the number of a tweet and $k$ represents the number of tokens in a tweet. At our deep intelligent contextual embedding layer we concatenated contextual embedding (ELMo), Word Embeddings (GloVe), Part of speech (POS) Embedding and Lexicon embedding. Detail of each embedding is given in the following sections.

1) Contextual Embedding: Words representation quality is measured by how it adds syntax information and handle polysemy into a model, which improves semantic word representation. Deep contextual embeddings [20] are embeddings proposed from language model (ELMo) which considers different aspects of words according to its usage in the context.

ELMo embeddings is based on the representation learned from Bi language model (BiLM). Log-likelihood of sentences in both forward and backward language models is involved in training process of BiLMs and final vector is computed after the concatenation of hidden representations from forward langugage model $\vec{h}_{n, j}^{L M}$ and backward langugage model $\overleftarrow{h}_{n, j}^{L M}$, where $j=1, \ldots ., L$ and is given by equation 1 .

$$
\begin{aligned}
B i L M= & \sum_{\substack{n=1 \\
k}}\left(\log p\left(t_{n} \mid t_{1}, \ldots ., t_{n-1} ; \Theta_{x}, \vec{\Theta}_{L S T M}, \Theta_{s}\right)\right. \\
& +\log p\left(t_{n} \mid t_{n+1}, \ldots ., t_{n} ; \Theta_{x}, \overleftarrow{\Theta}_{L S T M}, \Theta_{s}\right)
\end{aligned}
$$

\footnotetext{
${ }^{1} \mathrm{http} / / /$ sentiment.christopherpotts.net/code-data/happyfuntokenizing.py
}

where $\theta_{x}$ and $\theta_{s}$ are the token representation parameters and softmax parameters respectively which are shared between forward and backward directions. And $\vec{\Theta}_{L S T M}$ and $\bar{\Theta}_{L S T M}$ are the forwared back backward LSTM parameters respectively. ELMo abstracts the representations learned from intermediate layer from BiLM and execute linear combination for each token in a downstream task. BiLM contains $2 L+1$ set representations as given below.

$$
\begin{gathered}
R_{n}=\left(X_{x}^{L M}, \vec{h}_{n, j}^{L M}, \overleftarrow{h}_{n, j}^{L M} \mid \quad j=1, \ldots, L\right) \\
=\left(h_{n, j}^{L M} \mid \quad j=0, \ldots, L\right)
\end{gathered}
$$

where $h_{n, 0}^{L M}=x_{n}^{L M}$ is the layer of token and $h_{n, j}^{L M}=$ $\left[\vec{h}_{n, j}^{L M}, \overleftarrow{h}_{n, j}^{L M}\right]$ for each bi directional LSTM layer. ELMo is a task specific combination of these features where all layers in $M$ are flattened by ELMo into a single vector and is given by equation 2 .

$$
E L M o_{n}^{\text {task }}=E\left(M_{n} ; \Theta^{\text {task }}\right)=\gamma^{\text {task }} \sum_{j=0}^{L} s_{j}^{\text {task }} h_{h, j}^{L M}
$$

where $s^{\text {task }}$ are weights which are softmax normalized for the combination of different layers representations and $\gamma^{\text {task }}$ is a hyper parameter for optimization and scaling of Elmo representation. Our architecture is based on a pre-trained ELMo embeddings with a 1,024 dimensions obtained using the 1 Billion Word Benchmark which contains about $800 \mathrm{M}$ tokens of news crawl data from WMT 2011 [4]. ELMo gives us context Vector, $\mathbf{V}_{\text {context }}$ of 1024 dimensions, which has the polysemy and syntax information of tweets context.

2) GloVe Embedding: GloVe is an unsupervised learning model for obtaining word vector representations by aggregating global word-word co-occurrence statistics which counts how frequently a word appears in a context. GloVe uses ratios of co-occurrence probabilities.

As recommended by Peters et al. [20] that it is favourable to concatenate ELMo embeddings with traditional word embeddings such as Word2Vec and GloVe. In our model we have used pre-trained GloVe embedding of 300 dimensions which are trained on 840 billion token from common crawl because it gives better results as compared to Word2Vec in our case. GloVe outputs a vector, $\mathbf{V}_{G l o V e}$ of 300 dimensions, which has word semantics information of tweets context.

3) Part of Speech (POS) Embedding: POS tagging is an important step in which each word in the context is assigned with the appropriate POS tag. Using POS has shown good results in NLP related tasks. POS gives us the useful information about a word, neighbors and different syntactic categories of words such as verbs, nouns, adverbs and adjectives etc. In our proposed model we have used stanford parser for POS 
tagging which generates POS tags. Each POS tagged token is then transformed to a vector, $\mathbf{V}_{P O S}$ of 50 dimensions.

4) Lexicon Embedding: The lexicon based embedding is based on the extraction of sentiment scores from sentiment lexicon which is a list of words, specific terms and phrases. Using these lexicons can be useful in analyzing the text for sentiment analysis. Each lexicon contains a pair of word-sentiment where each words has its sentiment score which is between -1 to 1 , where value less than 0 represent negative words and positive for values above 0 . There are many sentiment and emotion lexicons resources are available so it is very important to select a right one or appropriate combination of lexicons. We selected a combination of 6 different lexicons after experimenting with different lexicons for extracting sentiments in our lexicon embedding. If any token is not available in any of these lexicons then we assigned a score of zero to that token. Our lexicon embedding outputs a vector, $\mathbf{V}_{\text {Lexicon }}$ of 6 dimensions.

We have used following six Lexicons in our model.

1) SenticNet 5.0 [3]

2) VADER [11]

3) Bing Liu Opinion Lexicon [10]

4) SemEval Twitter English Lexicon [18]

5) NRC Sentiment140 Lexicon [13]

6) Large-Scale Twitter-Specific Sentiment Lexicon [24].

After the creation four vectors individually, we concatenated all of them to get one vector $\mathbf{V}_{D I C E}$, which is clean and contains polysemy, word semantics, syntax and sentiment knowledge of words in a tweet and given by equation 3 .

$$
\mathbf{V}_{\text {DICE }}=V_{\text {context }} \oplus \mathbf{V}_{\text {GloVe }} \oplus \mathbf{V}_{\text {POS }} \oplus \mathbf{V}_{\text {Lexicon }}
$$

where element-wise symbol $\oplus$ in equation 3 denotes concatenation of all four vectors.

\section{BiLSTM Layer}

We have placed BiLSTM layer [22] on top of our DICE with attention layer for sentiment analysis to capture the information from both directions. A BiLSTM takes an input of a vector $\mathbf{V}_{D I C E}$ with a sequence of $x_{z}$ tokens and produces hidden representation $h_{i}$ at a given time $i$ by concatenating the hidden representations from both forward $\overrightarrow{h_{i}}$ and backward $\overleftarrow{h_{i}}$ LSTM and is given by equation 4 .

$$
h_{i}=\left[\overrightarrow{h_{i}} \| \overleftarrow{h_{i}}\right]
$$

where $\|$ in equation 4 , denotes the concatenation of outputs from both forward and backward LSTM.

\section{Attention layer}

Not all words play an equal role in understanding the meaning of the sentence. We used attention mechanism [25] to enforce the contribution of important words. Attention assigns a weight $a_{i}$ to each token through a softmax function and finally, representation $\mathbf{R}$ which is a weighted sum of all tokens is calculated and given by equation 5 .
TABLE I

SUMMARY OF ALL PARAMETERS

\begin{tabular}{cc}
\hline Name & Details \\
\hline Loss function & Binary Cross Entropy \\
\hline DICE dimension & 1380 \\
\hline Train-Test Split & $0.80-0.20$ \\
\hline Optimizer & Adam \\
\hline Learning Rate & 0.001 \\
\hline Back-Propagation & ReLu \\
\hline batch Size & 128 \\
\hline Dropout & 0.25 \\
\hline$L_{2}$ Regularization & 0.0001 \\
\hline Hidden Layer Dimension & 150 each \\
\hline Gausian Noise & $\sigma=0.3$ \\
\hline
\end{tabular}

$$
R=\sum_{i=1}^{z} a_{i} h_{i}
$$

where,

$$
\begin{gathered}
a_{i}=\frac{\exp \left(e_{i}\right)}{\sum_{t=1}^{z} \exp \left(e_{t}\right)}, \quad \sum_{i=1}^{z} a_{i}=1 \\
e_{i}=\tanh \left(W_{h} h_{i}+b_{h}\right)
\end{gathered}
$$

where $W_{h}$ and $b_{h}$ are learned parameters, $h_{i}$ is the concatenation of the representations of the forward and backward LSTM, introduced in equation 4.

\section{E. Output Layer}

We used representation $\mathbf{R}$ generated from an attention layer and fed to fully connected softmax layer to get the class probability distribution. We minimized binary Cross-entropy loss function $\mathbf{L}$ in which loss increases as the predicted probability $\mathbf{p}$ diverges from the actual label $\mathbf{y}$, is given by equation 6. Summary of all parameters is given in Table I.

$$
L=-(y \log (p)+(1-y) \log (1-p))
$$

\section{EXPERIMENTAL RESULTS}

In this section, we describe the datasets and experimental evaluations to show the effectiveness of our proposed model.

\section{A. Datasets}

We have used three airline related Twitter datasets, two of them crawled and labeled by us and one is publicly available. We chose airline related datasets because limited work has been done on airlines sentiment analysis using deep learning methods. We call our three datasets as Dataset 1, Dataset 2 and Dataset 3.

1) Dataset 1: This dataset is publicly available and taken from the Kaggle Datasets originally released by CrowdFlower. Total number of tweets given in the dataset is 14,640. Since we are dealing with binary classification problem so we are considering only positive and negative so after filtering the total number of tweets is 11,541 . The tweets are related to six major US Airlines: American airline, United airline, US Airways, Southwest airline, Delta airline, and Virgin airline. 
TABLE II

TWEETS DISTRIBUTION IN ALL DATASETS

\begin{tabular}{cccc}
\hline Dataset Name & Positive & Negative & Total \\
\hline Dataset 1 & 2363 & 9178 & 11541 \\
\hline Dataset 2 & 11670 & 4784 & 16454 \\
\hline Dataset 3 & 17860 & 4312 & 22172 \\
\hline
\end{tabular}

2) Dataset 2: The data was collected by using Tweepy, an official python Twitter API library. Dataset contains only two months worth of tweets starting from December 2015 until January 2016. We followed guidelines by Mohammad et al. in [17] for annotations of tweets and instructed annotators to label tweets as positive and negative. First, a set of 200 tweets were given, four different annotators annotated the tweets collectively so that all of them can have a general understanding and agreement on the standard for annotation. We used Cohens Kappa $(\kappa)$ for calculating inter annotator agreement (IAA) between annotators. In the second phase a same set of random 1000 tweets were given to each annotator for annotation. Disagreement was observed and resolved. In the third phase, again another same set of 500 tweets were given to all annotators and this time we achieved good IAA score and minor disagreement was observed. Finally in our last phase, large set of remaining tweets were equally divided among all annotators for annotation of tweets. Total number of tweets in dataset 2 are 16,454 . The tweets are related to three airlines in dataset 2 which are Cathay Pacific, United airline and Singapore airline.

3) Dataset 3: We used the same method to collect and annotate our third dataset as stated above. Total number of tweets given in our dataset 3 are 22,172. Dataset 3 contains tweets related to Emirates airline.

The distribution of all three datasets are given in Table II.

\section{B. Performance Evaluation}

1) Baselines: As a baseline, we compared the performance of our model with approaches mentioned in [9] and [6] but we used TF-IDF for features extraction instead of unigram, bi-gram and their combination for different classifiers and their ensemble. We have also compared our model with (i) deep convolutional neural network ${ }^{2}$ (DCNN) (embeddings initialized with GloVe word embeddings) [12] and (ii) CharSCNN/SCNN ${ }^{3}$ [7] (embeddings initialized with character (CharSCNN) and Word2Vec (SCNN) embeddings) where resulting embeddings are fed to deep neural networks. We have also compared our model with some recently proposed sentiment embeddings such as (i) hybrid ranking [23] which incorporates sentiment and context of tweets for Twitter sentiment analysis (HyRank ${ }^{4}$ ) and (ii) refined embeddings $\operatorname{Re}(*)$ [26] where researchers refined the traditional word embeddings by using intensity score from lexicon. Finally, we have compared our model with improved word vectors (IWV)[21] where traditional pre-trained word embeddings were enhanced by adding POS and sentiment information from

\footnotetext{
${ }^{2}$ https://nlp.stanford.edu/projects/glove/

${ }^{3}$ https://code.google.com/archive/p/word2vec/

${ }^{4}$ http://ir.hit.edu.cn/dytang/
}

TABLE III

COMPARISON OF DIFFERENT MODELS WITH PROPOSED MODEL

\begin{tabular}{|c|c|c|c|}
\hline Model & Dataset 1 & Dataset 2 & Dataset 3 \\
\hline TF-IDF SVM & .792 & .781 & .808 \\
\hline TF-IDF Naive Bayes & .831 & .836 & .827 \\
\hline TF-IDF Decision Tree & .861 & .853 & .876 \\
\hline TF-IDF Random Forest & .871 & .874 & .901 \\
\hline Lexicon Based Classifier & .624 & .715 & .691 \\
\hline Ensemble $(\mathrm{NB}+\mathrm{SVM}+\mathrm{DT}+\mathrm{RF})$ & $\overline{.816}$ & $\overline{.831}$ & $\overline{.829}$ \\
\hline Ensemble + Lexicon Classifier & .825 & .839 & .838 \\
\hline DCNN & .839 & .846 & .853 \\
\hline CharSCNN (Pre-trained) & .865 & .862 & .875 \\
\hline SCNN (Pre-trained) & .836 & .842 & .861 \\
\hline CharSCNN (Random) & .810 & .821 & .839 \\
\hline SCNN (Random) & .820 & .830 & .847 \\
\hline HyRank-BiLSTM & .848 & .846 & .868 \\
\hline $\operatorname{Re}($ Word2Vec)-BiLSTM & .853 & .852 & .872 \\
\hline Re(GloVe)-BiLSTM & .860 & .859 & .875 \\
\hline IWV & .884 & .875 & .890 \\
\hline$\overline{\overline{\text { DICE }}}$ & $\overline{.936}$ & $\overline{\overline{.931}}$ & $\overline{.939}$ \\
\hline$\Delta$ compared to previous best (IWV) & $5.88 \%$ & $6.40 \%$ & $5.50 \%$ \\
\hline
\end{tabular}

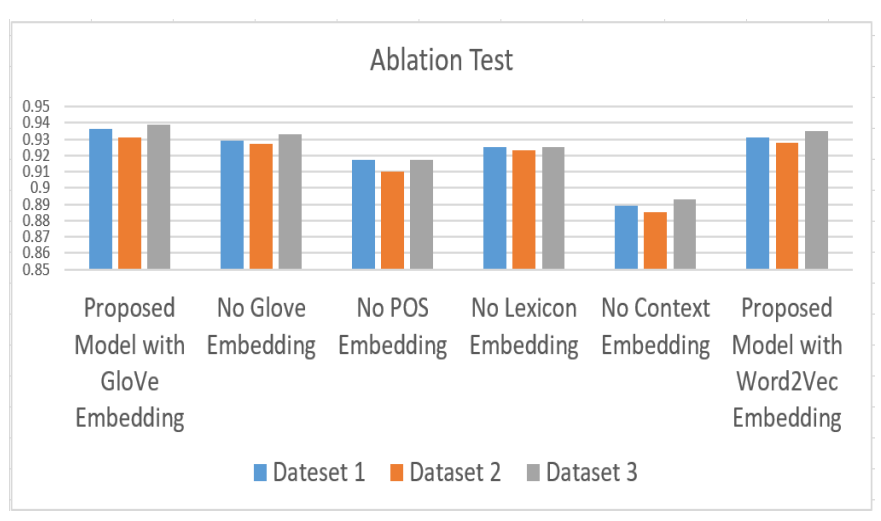

Fig. 4. Ablation Test of proposed model

lexicons for sentiment analysis. We selected those methods because they are the state-of-the-art ones and based on the conducted meta-analysis they exhibit the highest accuracy among the techniques developed so far.

2) Results: Accuracy results of our model are given in Table III. As we can see that the accuracy of our model is better than existing methods for sentiment analysis when testing them on three, airline related Twitter datasets. Our model achieved better performance because it improves quality of tweets by handling noise, polysemy, semantics, syntax and sentiments within tweet context. DICE improved the accuracy ratio $(\Delta)$ by $5.88 \%, 6.4 \%$ and $5.50 \%$ when compared to previous best results of embedding based IWV method and by $50 \%, 30.20 \%$ and $35.89 \%$ when compared to lowest results of lexicon based classier on datatset 1 , dataset 2 and dataset 3 respectively. As our model offers consistent improvement over all other methods for all tested datasets we can conclude that it is a robust solution for sentiment analysis problem.

3) Ablation Test: It is evident from Fig.4 that all embedding layers in our proposed model adds to the overall performance. The performance drops slightly for all datasets in both cases when we replace GloVe embedding with Word2Vec embed- 


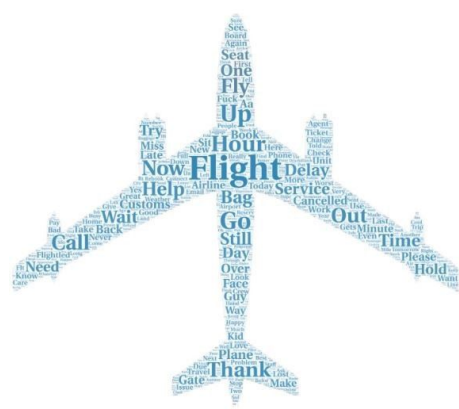

c)

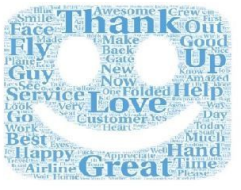

a)

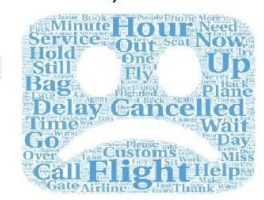

b)
Fig. 5. Word Cloud of a) Positive, b) Negative and c) All Tweets

ding or remove GloVe embedding from our model. Further, experimental analysis also indicates that performance drops in both cases when we remove POS and lexicon embeddings from our model and noticeable drop is observed when we remove context embeddings from our model. Hence, we can conclude that one of the strengths of our model lays in the combination of different components that builds the diversity which contributes to increased accuracy of sentiment classification. The word cloud of most common words in a) Positive, b) Negative and c) All Tweets are shown in Fig. 5.

\section{CONCLUSION}

In this paper, we proposed a Deep Intelligent Contextual Embedding model, DICE, which handles complicated attributes of words and its usage within the noisy tweet context. The proposed method handles the issues of polysemy, semantics, sentiment and syntax within the tweet context by learning representations from four different embeddings and our intelligent pre-processor compliments our model by removing the noise of informal and unstructured tweets. Additionally, we fed our DICE to BiLSTM with attention mechanism. The experiment shows that our model outperforms different baselines based on traditional word embeddings and sentiment embeddings for sentiment analysis of airline related tweets. In future, we plan to extend our model to character level and examine combination of embeddings hierarchically for social media sentiment anlysis. This will help to capture more features to improve accuracy.

\section{REFERENCES}

[1] Yoshua Bengio. Deep learning of representations: Looking forward. CoRR, abs/1305.0445, 2013.

[2] Erik Cambria, Soujanya Poria, Devamanyu Hazarika, and Kenneth Kwok. Senticnet 5: Discovering conceptual primitives for sentiment analysis by means of context embeddings. In AAAI, 2018.

[3] Erik Cambria, Soujanya Poria, Devamanyu Hazarika, and Kenneth Kwok. Senticnet 5: Discovering conceptual primitives for sentiment analysis by means of context embeddings. In AAAI, 2018.

[4] Ciprian Chelba, Tomas Mikolov, Mike Schuster, Qi Ge, Thorsten Brants, and Phillipp Koehn. One billion word benchmark for measuring progress in statistical language modeling. CoRR, abs/1312.3005, 2013.

[5] Franco Chiavetta, Giosu Lo Bosco, and Giovanni Pilato. A lexiconbased approach for sentiment classification of amazon books reviews in italian language. pages 159-170, 012016.
[6] Nádia F.F. da Silva, Eduardo R. Hruschka, and Estevam R. Hruschka. Tweet sentiment analysis with classifier ensembles. Decis. Support Syst., 66(C):170-179, October 2014.

[7] Cícero Nogueira dos Santos and Maíra A. de C. Gatti. Deep convolutional neural networks for sentiment analysis of short texts. In COLING, 2014.

[8] Kevin Gimpel, Nathan Schneider, Dipanjan Das, Daniel Mills, Jacob Eisenstein, Michael Heilman, Dani Yogatama, Jeffrey Flanigan, and Noah A. Smith. Part-of-speech tagging for twitter: Annotation, features, and experiments.

[9] Alec Go, Richa Bhayani, and Lei Huang. Twitter sentiment classification using distant supervision. Processing, pages 1-6, 2009.

[10] Minqing $\mathrm{Hu}$ and Bing Liu. Mining and summarizing customer reviews. In Proceedings of the Tenth ACM SIGKDD International Conference on Knowledge Discovery and Data Mining, KDD '04, pages 168-177, New York, NY, USA, 2004. ACM.

[11] Clayton J. Hutto and Eric Gilbert. Vader: A parsimonious rule-based model for sentiment analysis of social media text. In ICWSM, 2014.

[12] Zhao Jianqiang and Gui Xiaolin. Deep convolution neural networks for twitter sentiment analysis. IEEE Access, PP:1-1, 012018.

[13] Svetlana Kiritchenko, Xiao-Dan Zhu, Colin Cherry, and Saif Mohammad. Nrc-canada-2014: Detecting aspects and sentiment in customer reviews. In SemEval@COLING, 2014.

[14] Pengfei Liu, Xipeng Qiu, and Xuanjing Huang. Learning contextsensitive word embeddings with neural tensor skip-gram model. In Proceedings of the 24th International Conference on Artificial Intelligence, IJCAI'15, pages 1284-1290. AAAI Press, 2015.

[15] Bryan McCann, James Bradbury, Caiming Xiong, and Richard Socher. Learned in translation: Contextualized word vectors. In NIPS, 2017.

[16] Tomas Mikolov, Ilya Sutskever, Kai Chen, Greg S Corrado, and Jeff Dean. Distributed representations of words and phrases and their compositionality. In C. J. C. Burges, L. Bottou, M. Welling, Z. Ghahramani, and K. Q. Weinberger, editors, Advances in Neural Information Processing Systems 26, pages 3111-3119. Curran Associates, Inc., 2013.

[17] Saif Mohammad. A practical guide to sentiment annotation: Challenges and solutions. In WASSA@NAACL-HLT, 2016.

[18] Saif Mohammad, Svetlana Kiritchenko, and Xiaodan Zhu. Nrc-canada: Building the state-of-the-art in sentiment analysis of tweets. In Second Joint Conference on Lexical and Computational Semantics (*SEM), Volume 2: Proceedings of the Seventh International Workshop on Semantic Evaluation (SemEval 2013), pages 321-327. Association for Computational Linguistics, 2013.

[19] Jeffrey Pennington, Richard Socher, and Christopher D. Manning. Glove: Global vectors for word representation. In In EMNLP, 2014.

[20] Matthew E. Peters, Mark Neumann, Mohit Iyyer, Matt Gardner, Christopher Clark, Kenton Lee, and Luke Zettlemoyer. Deep contextualized word representations. CoRR, abs/1802.05365, 2018.

[21] Seyed Mahdi Rezaeinia, Ali Ghodsi, and Rouhollah Rahmani. Improving the accuracy of pre-trained word embeddings for sentiment analysis. CoRR, abs/1711.08609, 2017.

[22] M. Schuster and K.K. Paliwal. Bidirectional recurrent neural networks. Trans. Sig. Proc., 45(11):2673-2681, November 1997.

[23] Duyu Tang, Furu Wei, Bing Qin, Nan Yang, Ting Liu, and Ming Zhou. Sentiment embeddings with applications to sentiment analysis. IEEE Trans. on Knowl. and Data Eng., 28(2):496-509, February 2016.

[24] Duyu Tang, Furu Wei, Bing Qin, Ming Zhou, and Ting Liu. Building large-scale twitter-specific sentiment lexicon : A representation learning approach. In Proceedings of COLING 2014, the 25th International Conference on Computational Linguistics: Technical Papers, pages 172-182. Dublin City University and Association for Computational Linguistics, 2014.

[25] Zichao Yang, Diyi Yang, Chris Dyer, Xiaodong He, Alexander J. Smola, and Eduard H. Hovy. Hierarchical attention networks for document classification. In HLT-NAACL, 2016.

[26] Liang-Chih Yu, Jin Wang, K. Robert Lai, and Xuejie Zhang. Refining word embeddings using intensity scores for sentiment analysis. IEEE/ACM Trans. Audio, Speech and Lang. Proc., 26(3):671-681, March 2018. 